Received: 23 August 2018

Accepted: 7 March 2019

Published online: 12 April 2019
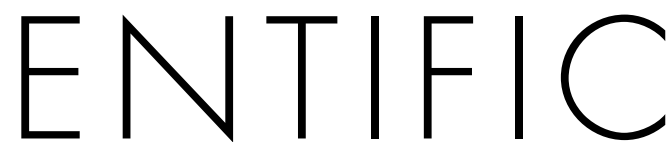

REP

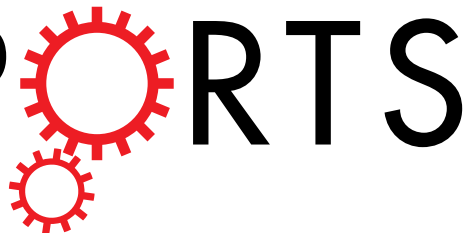

\title{
OPEN
}

\section{The development of a high-density genetic map significantly improves the quality of reference genome assemblies for rose}

Shubin $\mathrm{Li}^{1}$, Guoqian Yang ${ }^{2,6,7}$, Shuhua Yang ${ }^{3}$, Jeremy Just ${ }^{4}$, Huijun Yan ${ }^{1}$, Ningning Zhou ${ }^{1}$, Hongying Jian ${ }^{1}$, Qigang Wang ${ }^{1}$, Min Chen ${ }^{1}$, Xianqin Qiu ${ }^{1}$, Hao Zhang ${ }^{1}$, Xue Dong ${ }^{2}$, Xiaodong Jiang ${ }^{2,6}$, Yibo Sun ${ }^{2,6}$, Micai Zhong ${ }^{2,6}$, Mohammed Bendahmane $\mathbb{D}^{4}$, Guogui Ning ${ }^{5}$, Hong $\mathrm{Ge}^{3}$, Jin-Yong Hu $\mathbb{D}^{2}$ \& Kaixue Tang ${ }^{1}$

Roses are important woody plants featuring a set of important traits that cannot be investigated in traditional model plants. Here, we used the restriction-site associated DNA sequencing (RAD-seq) technology to develop a high-density linkage map of the backcross progeny (BC1F1) between Rosa chinensis 'Old Blush' (OB) and $R$. wichuraiana 'Basyes' Thornless' (BT). We obtained 643.63 million pair-end reads and identified 139,834 polymorphic tags that were distributed uniformly in the rose genome. 2,213 reliable markers were assigned to seven linkage groups (LGs). The length of the genetic map was $1,027.425 \mathrm{cM}$ in total with a mean distance of $0.96 \mathrm{cM}$ per marker locus. This new linkage map allowed anchoring an extra of 1.21/23.14 $\mathrm{Mb}(12.18 / 44.52 \%)$ of the unassembled OB scaffolds to the seven reference pseudo-chromosomes, thus significantly improved the quality of assembly of OB reference genome. We demonstrate that, while this new linkage map shares high collinearity level with strawberry genome, it also features two chromosomal rearrangements, indicating its usefulness as a resource for understanding the evolutionary scenario among Rosaceae genomes. Together with the newly released genome sequences for $O B$, this linkage map will facilitate the identification of genetic components underpinning key agricultural and biological traits, hence should greatly advance the studies and breeding efforts of rose.

Rose (Rosa sp., Rosaceae) is one of the most important horticultural plants. Besides its high ornamental values as garden plant and cut flowers, it also provides key materials for production of essential oils used in perfume and cosmetic products, and for food and medical products ${ }^{1,2}$. Roses have been cultivated since antiquity but the breeding activity that led to the production of modern roses only really began in the $19^{\text {th }}$ century $y^{3}$. In the genus of Rosa, there are about 200 species, among which 95 species are found in China, and more than 35,000 commercial cultivars (http://www.efloras.org). Most of the modern roses have a long and complex history of hybridization/ crossing and polyploidization process among a dozen species ${ }^{4-7}$. Due to high frequency of backcrossing with Asian germplasms, cultivated roses display a shifting pattern from European to Asian genetic backgrounds ${ }^{8,9}$. Besides their economical importance, roses feature key biological traits such as scent production, continuous

${ }^{1}$ National Engineering Research Center For Ornamental Horticulture, Flower Research Institute, Yunnan Academy of Agricultural Sciences; Yunnan Flower Breeding Key Lab, Kunming, 650231, China. ${ }^{2}$ CAS Key Laboratory for Plant Diversity and Biogeography of East Asia, Kunming Institute of Botany, Chinese Academy of Sciences, Kunming, 650201, China. ${ }^{3}$ Institute of Vegetables and Flowers, Chinese Academy of Agricultural Sciences, Beijing, 100081, China. ${ }^{4}$ Laboratoire Reproduction et Développement des Plantes, Univ Lyon, ENS de Lyon, UCB Lyon 1, CNRS, INRA, F-69364, Lyon, France. ${ }^{5}$ Key laboratory of Horticultural Plant Biology, Ministry of Education, College of Horticulture \& Forestry Sciences, Huazhong Agricultural University, Wuhan, 430070, China. ${ }^{6}$ Kunming College of Life Sciences, University of Chinese Academy of Sciences, Kunming, 650201, Yunnan Province, China. 7 Germplasm Bank of Wild Species, Kunming Institute of Botany, Chinese Academy of Sciences, Kunming, 650201, China. Shubin Li, Guoqian Yang and Shuhua Yang contributed equally. Correspondence and requests for materials should be addressed to H.G. (email: gehong@caas.cn) or J.-Y.H. (email: hujinyong@mail.kib.ac.cn) or K.T. (email: kxtang@hotmail.com) 
flowering (CF) and double flowers. Rose is now becoming a model species for woody plants as it has a relatively small genome (approximately $560 \mathrm{Mb}$ ) whose sequence was very recently released ${ }^{6,10}$ and an established transgenic systems $\mathrm{s}^{7,11-14}$.

The market for high-quality roses demands the continuous development of new varieties with better performance, such as disease resistance and flower quality. Rose breeding often requires tedious crosses between species, while marker-assisted selection (MAS) holds great promise for faster breeding of rose cultivars by speeding up progeny screening ${ }^{15,16}$. A high-resolution genetic map could be useful for map based cloning of genes associated with traits of interest ${ }^{17}$, and to improve genome assembly and comparative genomics ${ }^{18}$. It will provide an opportunity to understand the genomic architecture and chromosomal rearrangements that occurred during Rosa speciation and domestication.

The first molecular genetic linkage map for rose was constructed mostly with RAPD and AFLP markers by using a diploid F1 population derived from Rosa multiflora hybrids with the double pseudo testcross strategy ${ }^{19}$. Since then, several genetic maps have been constructed for diploid and tetraploid roses using AFLP, RFLP, SSR and/or CAPS markers ${ }^{20-27}$. Meanwhile, several important traits like flower color ${ }^{19}$, presence of prickles on the stem $^{19,24}$, resistance to black-spot ${ }^{28,29}$ and powdery mildew ${ }^{30}$, and flowering time ${ }^{25,27}$, have been mapped on these genetic maps ${ }^{23,31}$. However, in roses, the low marker density, along with the high costs and the time and labor required to develop new markers, make most of these maps unsuitable for fine mapping of traits of interest and useless for breeding programs. Whole genome-wide DNA markers are required to construct high-resolution linkage maps. One important type of DNA markers is SNP (single nucleotide polymorphisms). Recently, one 68k EST-based WagRhSNP array was developed for roses and allowed expanding existing tetraploid rose maps ${ }^{10,32,33}$.

Next-generation sequencing (NGS) technology provides opportunities to genotype a large number of individuals and unravel large number of SNP markers at the same time without the need of a reference genome sequence $^{34,35}$. Two typical methods to develop genome-wide markers with NGS technology are genotyping by sequencing ${ }^{36}$ and restriction site-associated DNA sequencing (RAD-seq) ${ }^{37-39}$. RAD-seq utilizes the power of NGS platforms to generate high coverage of short tags adjacent to restriction sites, which can then be used to identify single nucleotide polymorphisms (SNPs) between genotypes. Recently, SNP-based consensus-maps were constructed by RAD-seq for diploid roses ${ }^{10,40}$. Though genotyping by sequencing has been proved to be successful in detection of bi-allelic SNP markers in both diploid and tetraploid roses, a high-density genetic map for founder species of modern roses remains necessary to facilitate QTL positioning, map based cloning and comparative genomics studies ${ }^{10,40,41}$

Rosa chinensis 'Old Blush' (OB) and R. wichuraiana 'Basye's Thornless' (BT) are among the founder genotypes during rose domestication. OB contributed several novel but important traits such as CF and tea scent ${ }^{7,8,42-46}$. OB and BT feature several contrast phenotypes like continuous flowering (CF), number of petals, etc ${ }^{42}$. Previously, the development of a backcross population between OB and BT (BC1F1) has allowed us to genetically identify loci controlling recessive traits, like CF trait ${ }^{42}$, while the comparisons between $\mathrm{OB}$ and $\mathrm{BT}$ transcriptomes together with other Rosacaeae plants provided us the opportunity to identify molecular features characterizing roses $^{47}$. In this study, we used RAD-seq to screen for SNP markers in this population. This allowed us to construct a high-density SNP-based genetic map. Our map comprised seven linkage groups with a total of 2213 high quality SNP markers and was integrated with the current genome assemblies to define the scaffolds order.

\section{Results}

RAD-seq library construction and sequencing. A total of four RAD-seq libraries from the two parents and their 152 offsprings were constructed and sequenced. Approximately $6.55 \mathrm{~Gb}(\mathrm{BT})$ and $7.24 \mathrm{~Gb}(\mathrm{OB})$ raw data were generated prior to any quality filtering for the parents, while $6.52 \mathrm{~Gb}$ (BT; 21.73 million reads) and $7.22 \mathrm{~Gb}$ (OB; 24.09 million reads) were kept after quality filtering (Supplemental Table S1). About 95.6\% (minimum 93.38\%) of reads had an average quality higher than Q20 (Q20 indicates a 1\% chance of error), indicating the high-quality of the data. For the 152 individuals, sequencing of the RAD libraries generated a total of $193.12 \mathrm{~Gb}$ raw data. Of the raw reads, an average of 3.55 million reads per individual were retained after removing the putatively duplicated reads and reads without intact $E c o$ RI cutting sites (average complete enzyme digestion ratio was $94.32 \%)$. After quality filtering, a total of $191.69 \mathrm{~Gb}$ clean data $(99.23 \%)$ were retained with an average of $1.07 \mathrm{~Gb}$ per individual, which ranged from 0.49 to $4.97 \mathrm{~Gb}$ (Supplemental Table S1; Fig. S1). Overall, our RAD-seq data showed a high Phred quality (Q20 $\geq 93.38 \%$, Q30 $\geq 86.76 \%$ ), a stable GC content ranging from $36.81 \%$ to $38.41 \%$ and a high digestion rate from $85.94 \%$ to $97.80 \%$.

SNP discovery and genotyping. The number of RAD tags detected in the male parent (BT) and female parent (OB) was 296,621 and 198,349, with an average depth (the average number of reads per tag) of 58.35 and 78.69 times, respectively (Supplemental Table S2). For the progeny individuals, the number of tags ranged from 110,760 to 243,497 with an average of 167,392 and a mean depth of 16.08 times (8.29 to 181.53 times) (Supplemental Table S2, Fig. S2). After merging alleles together, 357,174 tags were detected, among which 139,834 were polymorphic with a polymorphism rate of $39.15 \%$. A total of 593,497 SNPs were identified in the polymorphic tags with an average of 4 SNPs in each tag. These SNPs were then classified into transitions and transversions based on nucleotide substitution. Transitions (59.37\%) were the main type and about 1.46 times larger than the transversions type $(40.62 \%)$. The number of $\mathrm{A} / \mathrm{G}$ transitions was nearly equal to $\mathrm{C} / \mathrm{T}$ transitions; the numbers of $\mathrm{A} / \mathrm{C}, \mathrm{A} / \mathrm{T}$, and $\mathrm{G} / \mathrm{T}$ transversions were nearly equal, however, almost two times higher than $\mathrm{C} / \mathrm{G}$ transversions (Table 1).

High-resolution genetic map construction. We used a two-step strategy to construct the genetic map. First, a screen for markers that were present in at least 140 individuals (missing data ratio $<6 \%$ ) identified a total of 793 markers. JoinMap 4.0 and Lep-Map 2.0 softwares were used to build linkage groups after removing 277 


\begin{tabular}{|l|l|l|l|}
\hline Transition & Counts & Transversion & Counts \\
\hline G-A & $175644(29.59 \%)$ & A-T & $75808(12.77 \%)$ \\
\hline T-C & $176740(29.78 \%)$ & A-C & $62386(10.51 \%)$ \\
\hline & & G-T & $64681(10.90 \%)$ \\
\hline & & G-C & $38238(6.44 \%)$ \\
\hline
\end{tabular}

Table 1. Types of SNPs (transition/transversion) for double alleles loci.

\begin{tabular}{|l|l|l|l|l|l|}
\hline LGs & Nb. of markers & \multicolumn{1}{l|}{ map_size (cM) } & average gap_size (cM) & biggest gap_size (cM) & Nb. Of unique positions \\
\hline \multicolumn{7}{|l|}{ Version 1.0} & 77.132 & 0.41 & 14.863 & 188 \\
\hline LG1 & 188 & 61.572 & 0.79 & 3.948 & 79 \\
\hline LG2 & 79 & 83.007 & 0.86 & 4.117 & 97 \\
\hline LG3 & 97 & 79.18 & 1.58 & 9.696 & 51 \\
\hline LG4 & 51 & 80.221 & 3.09 & 8.071 & 27 \\
\hline LG5 & 27 & 35.885 & 1.71 & 4.174 & 22 \\
\hline LG6.1 & 22 & 6.631 & 0.14 & 1.001 & 48 \\
\hline LG6.2 & 49 & 68.676 & 1.81 & 4.808 & 39 \\
\hline LG7 & 39 & 492.304 & 1.29875 & 14.863 & 551 \\
\hline all & 552 & & & & \\
\hline Version 2.0 & 140.202 & 0.97 & 6.077 & 145 \\
\hline LG1 & 386 & 176.381 & 1.12 & 5.008 & 158 \\
\hline LG2 & 325 & 190.96 & 1.01 & 3.792 & 191 \\
\hline LG3 & 393 & 71.889 & 1.22 & 4.521 & 60 \\
\hline LG4.1 & 114 & 5.836 & 0.11 & 0.534 & 53 \\
\hline LG4.2 & 53 & 208.63 & 1.07 & 9.953 & 196 \\
\hline LG5 & 503 & 129.579 & 1.07 & 7.969 & 122 \\
\hline LG6 & 243 & 103.948 & 1.08 & 5.623 & 97 \\
\hline LG7 & 196 & 1027.425 & 0.96 & 9.953 & 1022 \\
\hline all & 2213 & & & \\
\hline
\end{tabular}

Table 2. Key statistics for the linkage groups (LGs) of Version 1.0 and Version 2.0 maps.

markers that were severely distorted $\left(\chi^{2}>30, \mathrm{P}<0.01\right)$. Three individuals were excluded during marker analysis and mapping due to excessive missing alleles. Obscure markers that were assigned to different locations of a linkage group were removed after comparing the marker location determined by the two programs, and then the genotype matrix of rest of the markers were re-ordered until the relative order between markers arranged by the two programs did not conflict. This map was designated as Version 1.0 (Table 2, Fig. 1a). The Version 1.0 genetic map spanned a total of $492.3 \mathrm{cM}$ with a total of 552 markers distributed on 7 linkage groups (LG6 was split into two parts), consistent with the haploid chromosome number $(n=7)$. Overall, each linkage group contained 79 markers that spanned an average length of $70.3 \mathrm{cM}$, with a mean marker interval of $1.3 \mathrm{cM}$. The number of mapped markers per linkage group varied from 22 markers on LG6 to 188 markers on LG1. The smallest linkage group was LG6, which contained 71 markers spanning a length of $42.52 \mathrm{cM}$. The largest linkage group was LG4, which had 100 markers and a length of $85.81 \mathrm{cM}$. The maximum gap size in each linkage group ranged from $1.00 \mathrm{cM}$ on LG6 to $14.86 \mathrm{cM}$ on LG1, with an average of $6.33 \mathrm{cM}$ (Table 2).

To increase the number of markers on the genetic map, we next decreased the stringency and screened for markers present in at least 122 individuals (missing data ratio $<18 \%$ ). A total of 3145 markers were retained. After removing 903 markers with severe distortion $\left(\chi^{2}>30, P<0.01\right)$ and 4 individuals with an excessive of missing alleles, Lep-Map 2.0 was used to construct the Version 2.0 of the rose genetic map. To refine Version 2.0 map, we compared it to Version 1.0. After removing markers with inconsistent orders, we recalculated the genotype file and obtained the final rose map Version 2.0 (Table 2, Fig. 1b). Version 2.0 map showed a high consistent order of markers with the Version 1.0 except for some outliers (Supplemental Figs S3, S4).

The total length of Version 2.0 was $1027.4 \mathrm{cM}$, with a total of 2213 markers (1022 unique positions). The average distance between adjacent markers was $0.96 \mathrm{cM}$. The number of markers mapped per LG ranged from 167 (LG4) to 503 (LG5), with an average of 316. Linkage groups vary widely in size, with an average length of $146.77 \mathrm{cM}$. The largest linkage group was LG5, with a length of $208.63 \mathrm{cM}$ and a mean marker interval of $1.07 \mathrm{cM}$, while the smallest linkage group was LG4, with a length of $77.72 \mathrm{cM}$ and an average distance of $0.687 \mathrm{cM}$ between adjacent markers. The maximum gap size in each linkage group ranged from $0.53 \mathrm{cM}$ on LG4 to $9.95 \mathrm{cM}$ on LG5 (Table 2). The LG4 linkage group was short and broken into two parts (Fig. 1b), which could be related to the fact that the middle part of LG4 is highly enriched for repeat sequences, and thus a low occurrence of restriction enzyme cutting sites was found. Location of clustered markers, some of which could associate with the centromeric regions, was prominent on linkage groups LG1, LG2, LG3, LG5, LG6, and LG7 (Supplementary Fig. S5). 
a

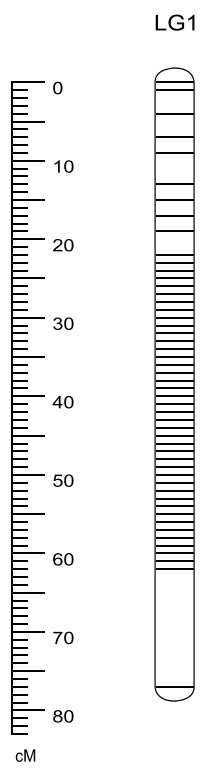

b

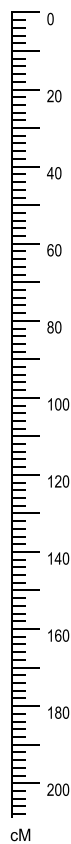

LG1
LG2

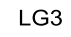

LG4

LG5

LG6.1

LG7
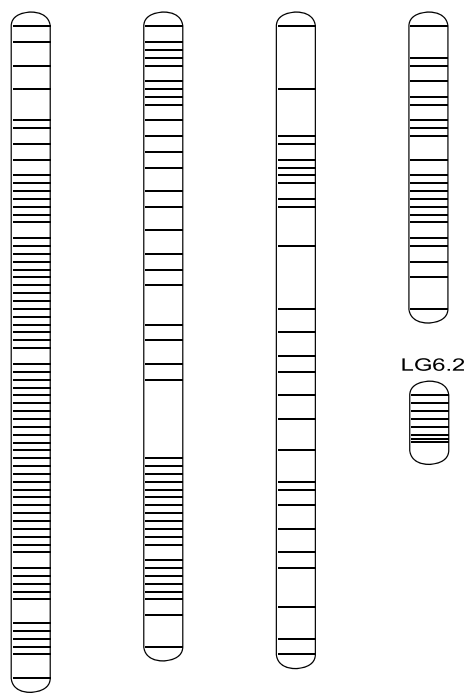

LG6.2
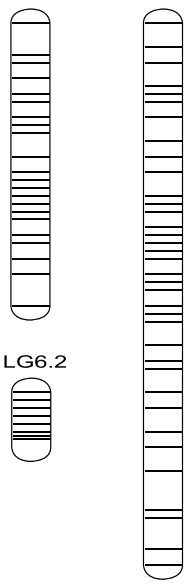

LG2

LG3

LG4.1

LG5

LG6

LG7
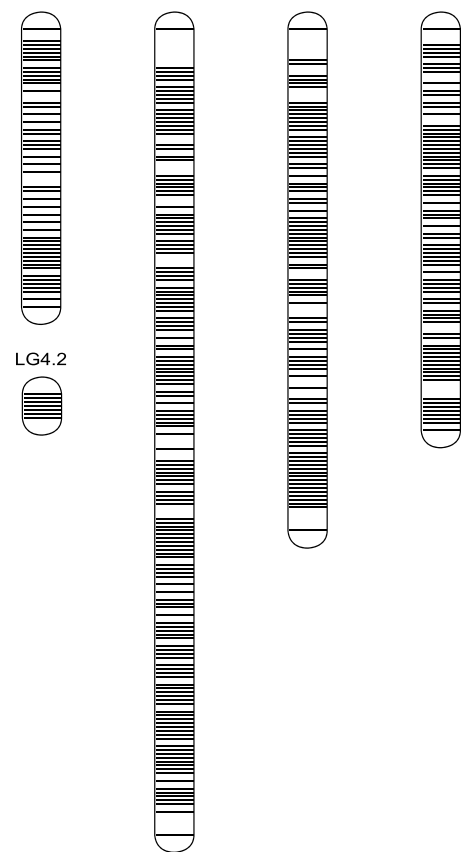

Figure 1. Linkage group length and marker distribution on the Version 1.0 genetic map (a) and Version 2.0 genetic map (b) of rose. Genetic map details are provided in Supplementary Table S2.

Marker names and positions for all SNP loci in the seven LGs of the genetic map were listed in Supplementary Table S4.

Distribution of distorted markers on the genetic map. In the Version 1.0 map, after the Chi-square test of genotypes, 607 markers $(76.54 \%, \mathrm{P}<0.01)$ severely deviated from the $1: 1$ segregation ratio, while 61 markers $(7.69 \%, 0.05<\mathrm{P}<0.01)$ deviated mildly from the $1: 1$ segregation ratio (a total account of $84.2 \%$ markers). In the Version 2.0 map, 2187 markers $(69.54 \%, \mathrm{P}<0.01)$ were found to be severely distorted from the $1: 1$ allele frequency, and 206 markers $(6.55 \%, 0.01<\mathrm{P}<0.05)$ were mildly deviated from 1:1 segregation after Chi-square test of genotypes, accounting for $76.09 \%$ of the total number of markers. We added distorted markers on the final map only when they did not affect the original orders of marker. To examine distribution of the distorted markers, we mapped all of them on the Version 1.0 map (Fig. 2). These markers were unevenly distributed along the seven LGs and tended to form 11 blocks of segregating distortion (RSD) on 6 LGs including 557 markers (Fig. 2; Supplementary Table S5). The smallest RSD block contained 8 markers $(1.7 \mathrm{cM})$, while the largest one contained 162 markers $(81.19 \mathrm{cM})$. LG1 contained the most RSDs, while LG7 contained the least distorted markers and no 


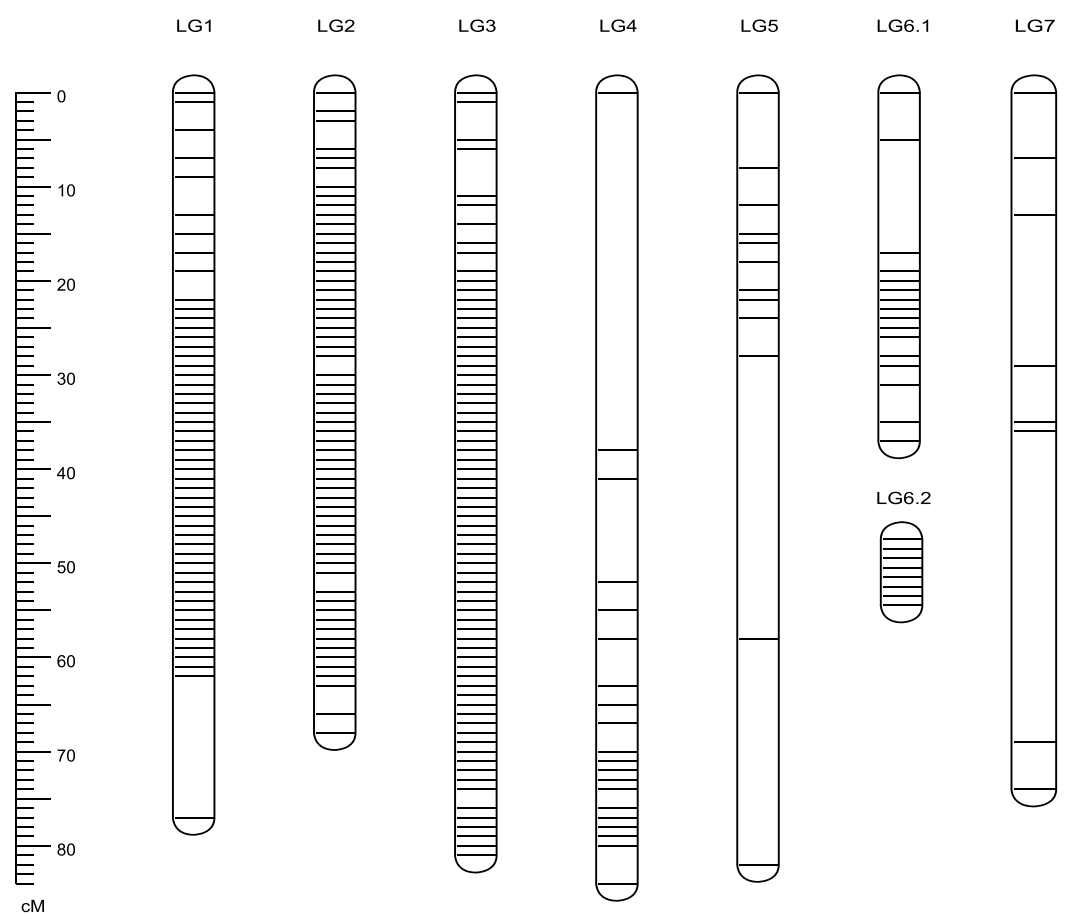

Figure 2. Distribution of distorted markers on the Version 1.0 rose genetic map.

RSD was found on LG7. Interestingly, the seven potential self-incompatibility-related genes (three encoding for S RNAses and four encoding S-locus type F-box proteins) were all found on the first RSD of LG1 $(17.14 \sim 23.59 \mathrm{cM}$, Chr3: $40570 \mathrm{~Kb} 40670 \mathrm{~Kb})^{10}$. There were 144 distorted markers caused by an excess of heterozygous alleles $\left(20.77 \%, \chi^{2}\right.$ test, $\left.\mathrm{P}<0.05\right)$, while the other 549 markers were due to excess of homozygous alleles $\left(79.22 \%, \chi^{2}\right.$ test, $\mathrm{P}<0.05$ ) (Supplementary Fig. S6).

Evaluation of the genetic map. We evaluated the Version 1.0 map with heat map (representing the recombination relationship between markers in each LG and identification of potential ordering errors) and with haplotype map (graphical genotypes, detecting the occurrence of double crossover events) assays. Heat maps showed that most of the 552 mapped-markers in most LGs were well ordered (Fig. 3). Consequently, graphical genotypes were generated based on these markers (Supplementary Fig. S7). From the color change indicates the occurrence of a recombination event we defined the majority of recombination blocks and identified relatively low proportion of double crossover and missing markers for each LG. Among the 754,284 contigs produced via clustering the $6 \mathrm{~Kb}$ library reads, seven pairs of adjacent markers that could hit the same contig were detected, evidencing the reliable order of these markers (Supplementary Table S6, Fig. S8). The final map covered more than $99.12 \%$ of the diploid rose genome.

Relationships between genetic and physical maps. To determine the relationships between our genetic map and the newly published physical map $^{6}$, marker sequences from Version 2.0 map were aligned to the OB genome. The OB doubled-haploid genome contains seven pseudomolecules that were built by integrating 25,695 markers of the K5 rose high-density genetic map. Using BLAST searches, a total of 1,371 RAD markers showed a unique match to the $R$. chinensis genome. Notably, most of the 7 linkage groups were syntenic with one of the 7 chromosomal pseudomolecules from the assembly, while only $<0.5 \%$ markers mapped onto linkage groups conflicted with their position in the assembled chromosomes (Fig. 4a). Chromosome segments with inversion or rearrangement were found at the end of LG1 and LG3. The discrepancy may be due to miscalculation, which occurs usually at both ends of a linkage group during linkage analysis. To clarify this situation, we compared the rose GBS genetic map published by Yan et al. ${ }^{40}$ with the published rose genome and detected similar rearrangements at the end of LG4, LG5, LG6 and LG7 (Fig. 4b). Furthermore, we observed a chromosome-segment-inversion between the middle of LG1 (30-40 cM) and the Chr3 of the genome. This inversion is still presence even if we use JoinMap to reorder this LG, indicating that it is not caused by random error during map construction. As the genome assembly is of high-quality and confirmed by mapping of Hi-C chromosomal-contact-map data ${ }^{6}$, this discrepancy might be due to genomic rearrangements between OB and BT, but not to scaffold misplacement which were usually found in some genome sequencing projects. The highly heterozygous $\mathrm{OB}$ genome could provide an alternative explanation for this discrepancy. However, we cannot exclude the possibility of genotyping errors due to low sequencing depth of some markers.

The RAD-seq based genetic map was further used to improve the OB genome assemblies ${ }^{6,10}$ (here denoted as the Raymond's and Hibrand Saint-Oyant's genomes, respectively). For the Raymond's genome, six additional previously unrecorded contigs $(1.21 \mathrm{Mb})$ were anchored to specific chromosomes, accounting for $12.18 \%$ of 


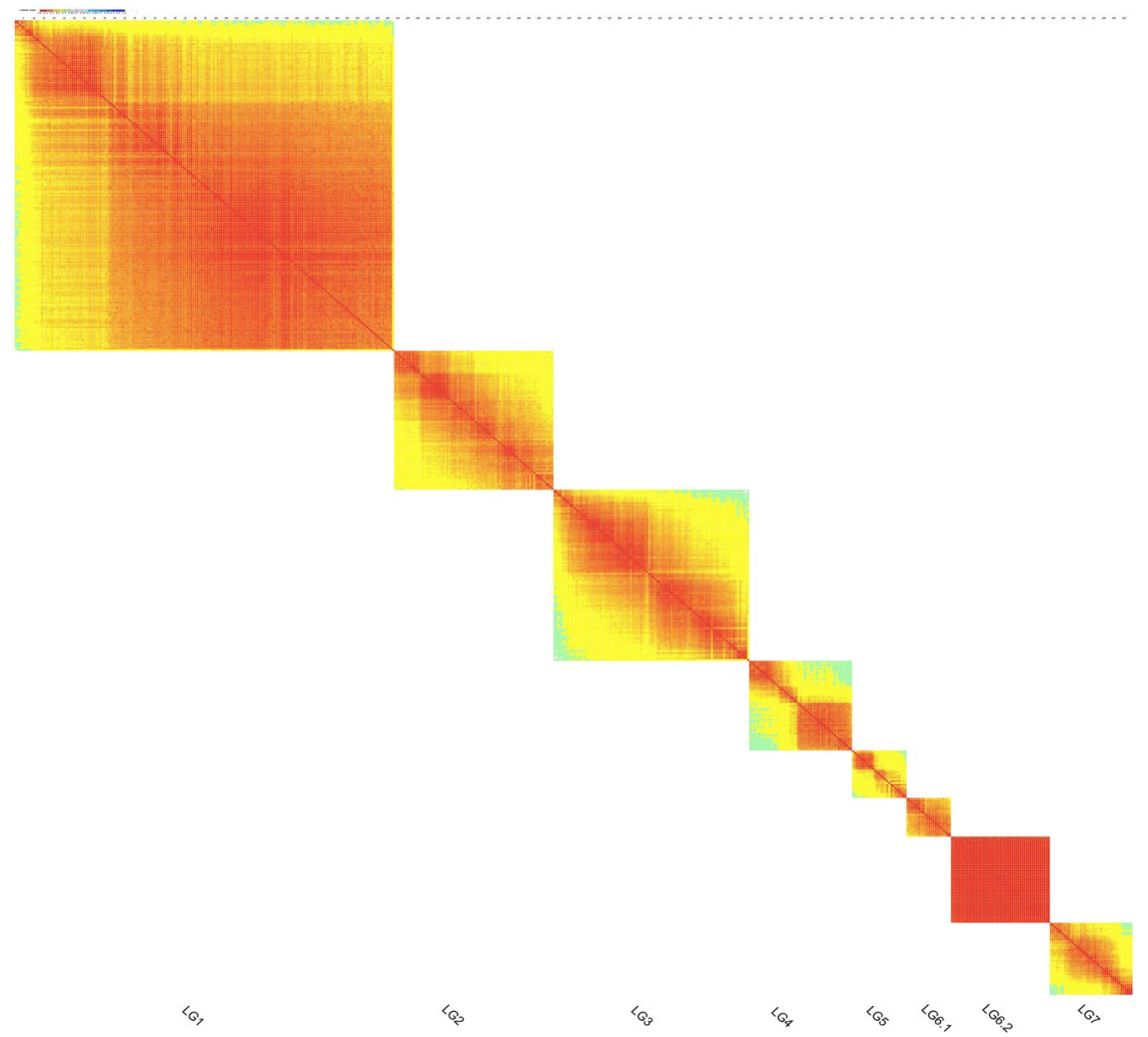

Figure 3. Heat maps reflecting the recombination relationship between markers in each linkage group. Each cell represents the recombination rate of two markers. Yellow color indicates a lower recombination rate while red color indicates higher recombination rate.

the unanchored contigs $(9.94 \mathrm{Mb})$. However, we were unable to determine their orientation on the chromosome because each contig contained only one marker. The list of newly anchored contigs to the LGs is presented in Table 3. We also identified 11 contigs containing a significant amount of centromeric repeats (Table 3, Supplementary Table S8). Circos plot of 7 pseudo-chromosomes with genetic (in cM) to physical (in Mb) distances is presented in Fig. 4. For the Hibrand Saint-Oyant's genome, about 1240 markers were anchored to the seven chromosomes, while about 103 hit to the unassigned contigs ( 44.52\%, 23.14 Mb of the $52 \mathrm{Mb} \mathrm{Chr})$ ). Among all the unassigned contigs, one contig was of chloroplast origin, eight were from mitochondria, and 231 were centromeric (Supplementary Table S9). No marker hits to the centromeric contigs. In general, these results reveal the potential of this map in improving rose genome assemblies (Supplementary Fig. S9; Table S7).

Synteny between rose and Fragaria vesca. Rosa sp. and Fragaria vesca (wild strawberry) exhibit high synteny ${ }^{6}$. Thus we investigated the correspondence between our newly constructed LGs and $F$. vesca chromosomes. About $13.75 \%$ (297) tags on our genetic map have homologs in F. vesca genome (v1.1 assembly). Among the 297 homologs, 287 were in highly syntenic regions and collinear with $F$. vesca chromosomal regions. An apparent 1:1 correspondence relationship between five $F$. vesca chromosomes $(2,3,4,5$, and 7$)$ and five of our rose LGs $(2,3,4,6$ and 7$)$ was observed with a good collinearity along the entire LGs. The remaining two LGs (1 and 5) showed chromosomal rearrangements (fusions and fissions) (Fig. 5, Supplementary Table S10). Rose LG1 shares synteny with half of Chr1 and half of Chr6 of strawberry, while LG5 features synteny with the remaining segments of strawberry Chr1 and Chr6 (Fig. 5). A further comparison with the newest version of $F$. vesca genome assembly (v4.0.a1) ${ }^{48}$ showed the same patterns (Supplementary Fig. 10). These differnces could be due to inter-chromosomal rearrangements after the divergence of the Fragaria and Rosa species from their last common ancestor. Instead, a comparative genomic study within the Rosaceae family showed that rose and strawberry originate from a common ancestral Rosoideae Karyotype (ARoK) through a chromosomes fusion for strawberry genome, whereas the rose went through one fission and two fusions, independently from strawberry ${ }^{6}$. Our pattern is thus consistent with these published paleogenomic results and also with other data based on the linkage map of diploid and tetraploid roses as well as the rose whole genome assemblies ${ }^{6,10,32,40}$.

\section{Discussion}

In this study, we used RAD-seq technology to construct a high-density linkage map with 139,834 polymorphic SNP tags and 2,213 markers between two rose genotypes and their BC1F1 progeny. The new linkage map contains seven LGs with a total length of $1,027.4 \mathrm{cM}$ and an average marker spacing of $0.96 \mathrm{cM}$. While all previously published maps were based on F1 mapping populations utilizing pseudo-testcross mapping strategy, this study 
a

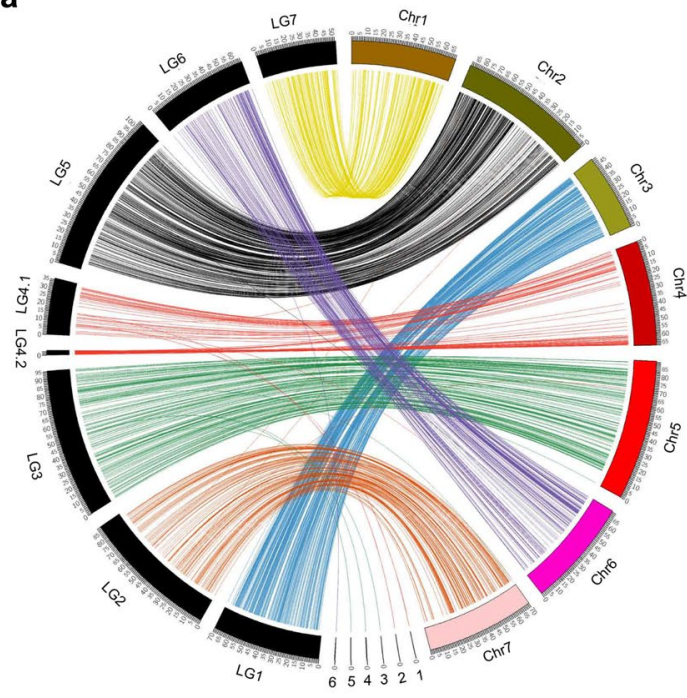

C

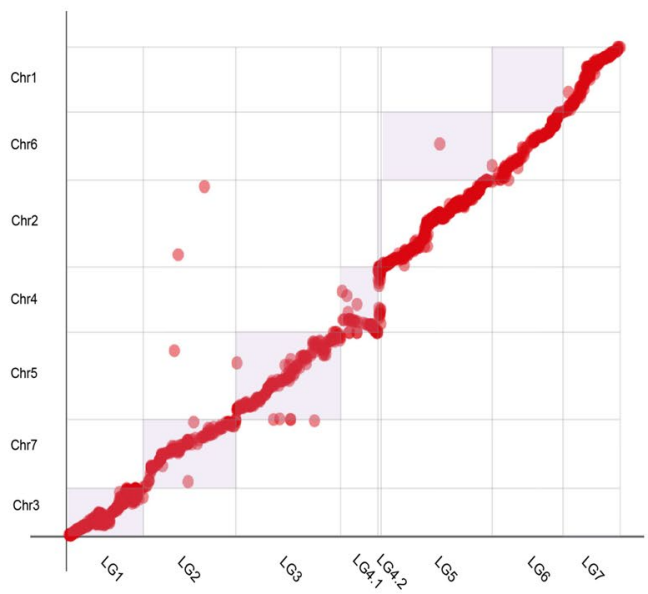

b

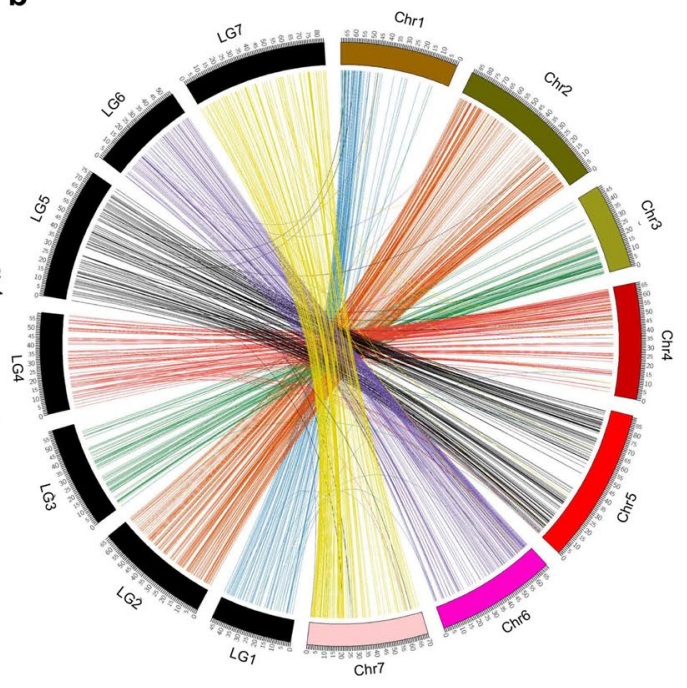

d

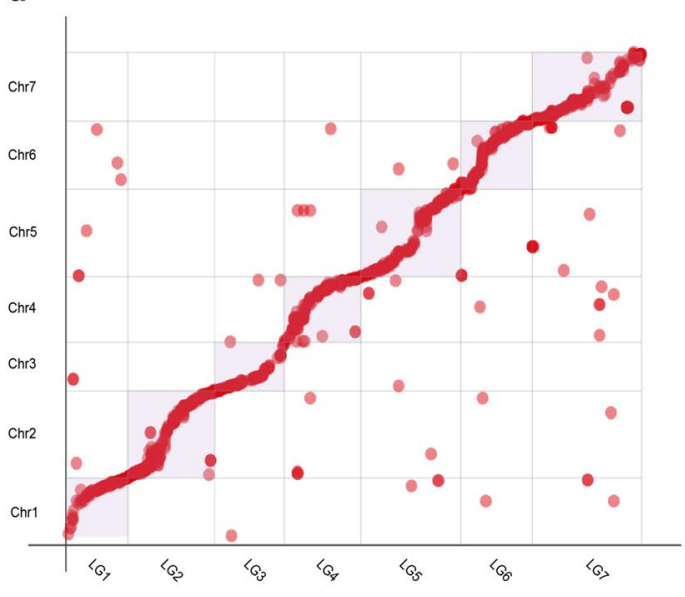

Figure 4. High conservation level of synteny between the constructed genetic map and the OB reference chromosomes $^{6}$. (a,c) Comparison of the syntenic relationship between the seven LGs (LG1-7) constructed in this research (in black) with the seven published OB reference Chromosomes (Chr1-7, in brown). 1-6 marks the unassigned and unordered contigs in OB reference genome. (b,d) Comparison of the syntenic relationship between seven LGs published by Yan et al. ${ }^{40}$ (in black) with the reference OB genomes (in brown). Chromosome 0-7 marks the published $\mathrm{OB}$ assembly.

represents the first rose map constructed by using RAD-seq on a $\mathrm{BC} 1 \mathrm{~F} 1$ population. This newly constructed map will be highly valuable for dissecting the molecular genetics of important trait, especially those with recessive inheritance nature ${ }^{42}$.

This map possesses two major advantages over previous maps. First, the map is of high density and high resolution. The 2,213 markers (1,022 unique positions) cover about $1,027.4 \mathrm{cM}$ on the seven LGs with an average marker density of 2.15 markers per cM. Therefore, this map represents an improvement over the integrated consensus map based on SSR and AFLP markers reported previously ${ }^{31}$, and offers rose geneticists and breeders a broader suite of options of marker selection for a wide range of research purposes. Secondly, all markers in this map are sequence-defined, and can be easily and unambiguously identified in any rose germplasm. These are very useful for comparative genomic studies with other plant species. It's worthy to note that markers in this map are not uniformly distributed on each LG (Fig. 1, Supplementary Fig. S5). We observed clustering of markers into short intervals in almost all LGs, which could be due to suppressed recombination at the telomeric and centromeric regions ${ }^{49,50}$. However, this clustering may also reflect the unequal distribution of EcoRI cutting sites in different chromosomal regions ${ }^{24}$. Despite the high-density of this RAD-seq based map, gaps still exist in most of the LGs. Indeed, there are more than 10 gaps exceeding $5 \mathrm{cM}$ with the largest gap covering $9.953 \mathrm{cM}$ on LG5. Identifying additional markers with a different enzyme or enzyme pairs (for example, AvaII $+M s p$ I) ${ }^{39}$ should help filling these large gaps.

We used a two-step strategy to generate this map for guaranteeing the quality and reliability. Parental linkage groups were first constructed using high quality markers with low missing ratio using simultaneously the JoinMap and Lep-Map programs with high LOD score (LOD $\geq 6.0$ ). Different algorithms for ordering markers in 


\begin{tabular}{|l|l|l|l|l|}
\hline LG & Marker & Location $(\mathbf{c M})$ & Scaffold & Location (bp) \\
\hline LG3 & 98870 & 68.563 & Chr0c37 (NW_020126846.1) & 61,300 \\
\hline LG3 & 200853 & 99.204 & Chr0c11 (NW_020126825.1) & 44,754 \\
\hline LG3 & 200854 & 99.866 & Chr0c21 (NW_020126834.1) & 132,452 \\
\hline LG4.1 & 192439 & 15.729 & Chr0c45 (NW_020126852.1) & 33,995 \\
\hline LG4.1 & 192439 & 15.729 & Chr0c45 (NW_020126852.1) & 93,608 \\
\hline LG4.1 & 51116 & 29.972 & Chr0c16 (NW_020126829.1) & 69,353 \\
\hline LG4.1 & 51116 & 29.972 & Chr0c16 (NW_020126829.1) & 45,922 \\
\hline LG6 & 234097 & 30.062 & Chr0c15 (NW_020126828.1) & 213,124 \\
\hline Centromere & & & Chr0c43 & \\
\hline Centromere & & & Chr0c24 (NW_020126837.1) & \\
\hline Centromere & & & Chr0c31 (NW_020126842.1) & \\
\hline Centromere & & & Chr0c08 (NW_020126822.1) & \\
\hline Centromere & & & Chr0c02 (NW_020126816.1) & \\
\hline Centromere & & & Chr0c18 (NW_020126831.1) & \\
\hline Centromere & & & Chr0c12 (NW_020126826.1) & \\
\hline Centromere & & & Chr0c14 & \\
\hline Centromere & & & Chr0c46 & \\
\hline Centromere & & & Chr0c42 & \\
\hline Centromere & & & Chr0c25 (NW_020126838.1) & \\
\hline
\end{tabular}

Table 3. List of the newly anchored scaffolds to the Raymond's genome ${ }^{6}$.

the two programs corroborated the accuracy of our rose genetic map Version 1.0. Next, we added, to this Version 1.0, markers with high missing ratio, while excluding those that may cause conflicting marker orders. The final map, resulting from several rounds of grouping, is more reliable than maps generated with genotypes of high missing ratio because of the presence of genotyping errors in markers and wrong estimates of recombination rates (Supplementary Fig. S4). Since both heat map and haplotype map confirmed that most markers were well ordered in all LGs, and the order of most of these markers on the map was consistent with that observed in the reference genome $^{6}$, we conclude that the newly developed integrated map is of high quality and reliability. Additionally, this newly constructed map is rather complete, though more markers are necessary to reinforce, for example LG4.1 and LG 4.2.

High frequency of segregation distortion (SD) is not unusual in outcrossing Rosaceae species ${ }^{50,51}$ like roses ${ }^{24,26}$. In Fragaria, 54\% of the polymorphic loci deviated from the expected 3:1 or 1:2:1 ratio in a F2 population. In rose F1 populations, it was observed that $28.9 \%$ of the markers in H190 (male parent) deviated significantly from the expected segregation ratio of $1: 1$, while $50 \%$ of the markers in $R$. wichuraiana (female parent) deviated significantly from the expected segregation of 1:1 ratios at $\mathrm{P}=0.005^{24}$. In the 93/1-119 (P119) X 93/1-117 (P117) F1 population, about $22 \%$ of distorted markers were found $(\mathrm{P} \leq 0.05)^{26}$. As for our newly constructed map, $84.2 \%$ markers departed from the expected 1:1 Mendelian segregation ratio in the Version 1.0 map, and $76.09 \%$ in Version 2.0 map $\left(\chi^{2}\right.$, $0.05<\mathrm{P}<0.01$ ). This pattern has ever been detected in a pummelo (Citrus grandis) F1 population ${ }^{52}$. One possible explanation could be that segregation distortion was brought in by non-randomized sampling and data filtering as well as insufficient sequencing depth. Insufficient sequencing could produce over-representation of one of the alles at the heterozygous locus and thus cause segregation distortion. At the same time, we cannot rule out the possibility that certain genotypes remain undetected because of incomplete digestion by the restriction enzyme. Another explanation can be that biological factors, e.g. gametophytic selection and lethal genes, could also cause segregation distortions $s^{24,26,51}$. Gametophytic selection by sub-lethal genes conferring "low viability" of zygote, embryo or seedling, could be located close to the distorted markers in maps. Presence of a self-incompatibility locus in the 93/1-119 (P119) x 93/1-117 (P117) F1 population and in R. rugosa has been reported ${ }^{26,53}$. 557 markers in our map were unevenly distributed along the seven LGs and formed 11 regions of segregating distortion (RSD) on 6 LGs including. Indeed, one RSD region harbored the potential self-incompatible loci (RNases and F-box proteins) ${ }^{10}$. However, the high level of synteny between our genetic maps and chromosomal-level physical maps ${ }^{6,10}$ indicates that the high proportion of distorted markers is more likely due to biological factors (genetic divergence between the parent plants) than to technical bias in the genotyping process.

Chromosome inversions were detected in rose genomes. Though our newly constructed map featured a good collinearity with the reference genome assembly, an inversion in the middle of LG1 (Chr3) was observed using both JoinMap and Lep-Map approaches. This inversion is not present in a rose consensus map (J06-20-14$3 \times$ 'Little Chief', J06-20-14-3 $\times$ 'Vineyard Song' and 'Old Blush' $\times$ 'Red Fairy') $)^{40}$. Distorted segregation of chromosome rearrangement among different species might explain this phenomenon ${ }^{54}$. Further studies with cytogenetic methods and a bigger mapping population should clarify this situation.

Genome architecture varies in roses. The presented genetic map revealed a high level of synteny conservation and genome rearrangements during evolution of roses and strawberry from their last common ancestor(s). The ancestral karyotype of Rosoideae (including strawberry and rose, RoAP) had eight protochromosomes ${ }^{6}$. Strawberry may have experienced an ancestral chromosome fusion from the eight protochromosomes to reach its current genome structure, while rose genome went through one independent fission and two fusions ${ }^{6}$. Both 
a

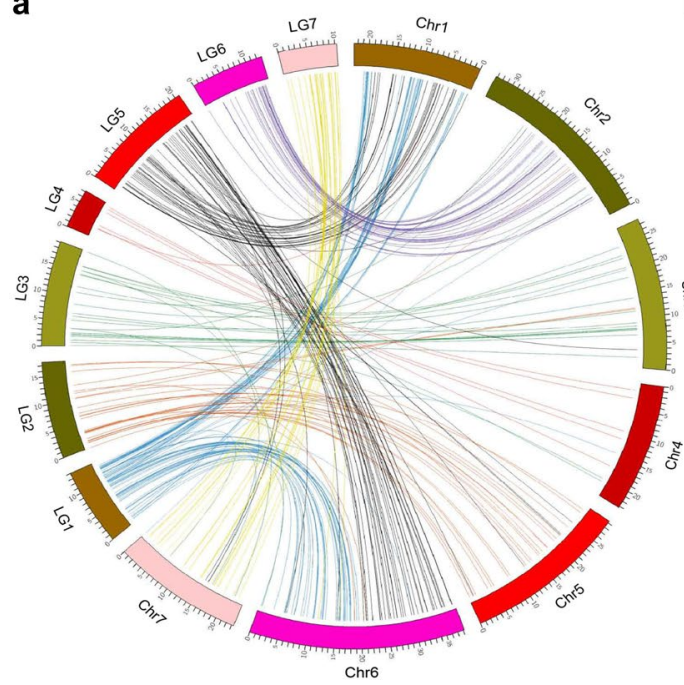

b

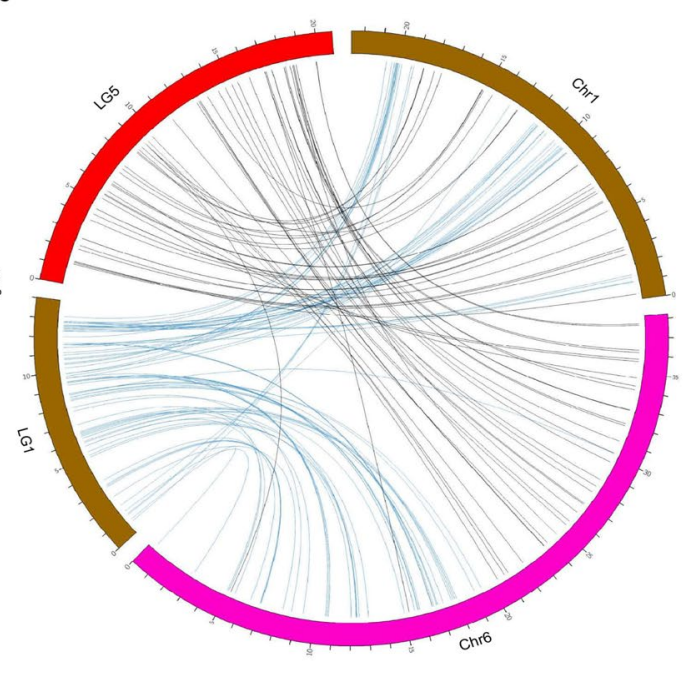

C

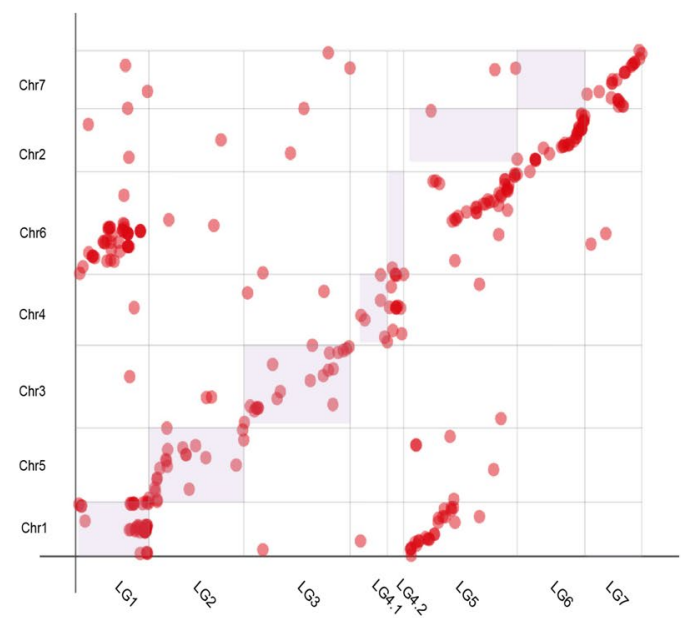

d

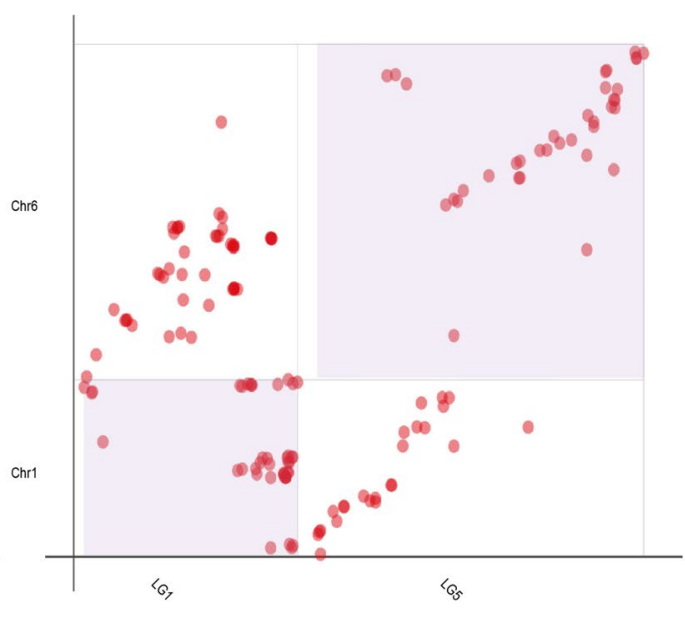

Figure 5. Comparison of the newly constructed linkage map and Fragaria vesca genome. (a,c) Comparison of the syntenic relationship between the LGs constructed in this study (LG1-7) with the seven F. vesca chromosomes (v1.1; Chr1-7). (b,d) Detailed comparison of the syntenic relationship between LG1 and LG5 with $F$. vesca Chr1 and Chr6 showing chromosomal rearrangements.

rose LG1 and LG5 are formed by a fusion of strawberry Chr1 + Chr6 fragments (Fig. 5). This is consistent with observations from whole genome sequencing and genetic maps of the diploid and tetraploid roses ${ }^{6,10,32,33,40}$. The potential breakpoints are close to predicted centromere positions, thus fits well with a telomere-centric model underlying karyotype evolution of most plants ${ }^{55}$. Genome diversity observed in this newly constructed genetic map and known maps as well as in the reference genome sequences should provide important information on genome evolution in Rosaceae.

The newly constructed map and the available reference genome sequences will greatly promote roses to become models for studying flowering behavior variation and other important traits in ornamental plant ${ }^{13}$. Indeed, this genetic map significantly improves the quality of reference genome assemblies. Thanks to the markers of this map, about $1.21 \mathrm{Mb}(12.18 \%)$ and $23.14 \mathrm{Mb}(44.52 \%)$ of the unassigned contigs could be anchored to the two recently published reference rose genomes generated from doubled-haploid OB plants ${ }^{6,10}$. As we excluded the markers with multiple hits to the unassigned contigs, no marker associated with unassigned centromeric-contigs was detected. Integration of this high-density linkage map with the assembled genomes should benefit significantly the dissection of molecular mechanisms underpinning key biological traits and thus improve the breeding of roses ${ }^{42}$.

\section{Materials and Methods}

Plant materials and DNA extraction. The BC1F1 population between Rosa chinensis 'Old Blush' (OB) and $R$. wichuraiana 'Basyes' Thornless' (BT) was developed as described previously ${ }^{42}$. Briefly, a F1 progeny was produced via crossing the vegetatively propagated OB and BT plants. Then the F1 plants with simple flowers (five petals only) were taken as the female plants and pollinated by pollens from OB. This process was repeated 
in 2013 and 2014 to generate a population of 152 BC1F1 individuals. This population segregates for at least six pairs of biological traits including continuous flowering and petal numbers. For genotyping and RAD-seq, young healthy leaves from two parents and BC1F1 individuals were collected. Genomic DNA was extracted with CTAB method $^{56}$ and quantified with a NanoDrop ND1000 spectrophotometer and Qubit 2.0 Fluorometer (Thermo Fisher Scientific, Rochester, NY, USA). DNA concentrations were adjusted to $50 \mathrm{ng} / \mu \mathrm{l}$ using Tris-EDTA buffer.

RAD library construction and high-throughput sequencing. RAD library construction, sample indexing and pooling were carried out as previously described ${ }^{37}$. DNA of two parents and each of their derived offspring was digested with EcoR I (New England Biolabs, Ipswich, MA, USA). Various P1 adapters, each with a unique 4-8 bp molecular-identifying sequence (MID, or barcode), were then ligated to designated individuals with T4 DNA ligase (New England Biolabs, Ipswich, MA, USA). The adapter-ligated products were then pooled in groups of 24 individuals and randomly sheared into DNA fragments. Sheared DNA was purified, eluted, and separated using gel electrophoresis, and DNA bands corresponding to 300-700 bp were excised and purified with magnetic beads. After end repair, purification, and elution, dATP overhangs were added to the DNA fragments. A paired-end P2 adapter containing T-overhangs was ligated to $20 \mu \mathrm{l}$ of sheared, size-selected, P1-ligated, and pooled DNA templates with a specific barcode. The ligated material was then purified, eluted, and subjected to PCR enrichment and sequencing on a HiSeq2500 next-generation sequencing platform (Illumina, San Diego, CA, USA) with PE150 mode. Sequencing data for each individual were then de-multiplexed according to the specific barcode and index. Length of raw reads was $142-146 \mathrm{bp}$ after removing barcode sequences. The original sequencing datasets have been deposited in the NCBI SRA repository with the accession number PRJNA516159.

Read processing and SNP identification. Raw reads were processed with the Stacks pipeline (version 1.41) to call SNPs ${ }^{57}$. Raw reads were firstly filtered to generate clean reads with the process_radtags program (parameters: $-\mathrm{r},-\mathrm{c},-\mathrm{q}$ ). Only the forward reads were kept for analysis as the reverse reads are at irregular distance from the restriction enzyme cutting site. To avoid artifacts, the following criteria were used to filter raw reads: (1) putative duplication reads generated by PCR amplification in library construction were discarded; (2) reads with adapter contamination were removed; (3) reads with $\geq 10 \%$ unidentified nucleotides (Ns) were discarded; (4) reads with average Pred quality score over a 15 bp sliding window below 10 ( $90 \%$ confidence) were removed; (5) reads without the correct partial $5 \mathrm{bp} E c o R I$ recognition sequence (AATTC) were discarded. Then the ustacks program was used to align clean read sequences into exactly-matching stacks (or alleles) and compared the stacks to form a set of putative loci using a maximum likelihood framework for each individual (parameters: $-m 3,-M$ 2). Loci that were two standard deviations above the mean depth of coverage were excluded, and then the cstacks program was used to merge loci of each parent into a catalog. The between-individual distance parameter of cstacks for mismatches was set 4 (parameter: $-n 4$ ). Then sstacks was run to match every individual in the population (including the two parents) against the catalog to identify locus/haplotype combinations in each individual. Finally, genotypes program was executed to call and export genotypes in a format compatible for JoinMap 4.0. The minimum number of matching progeny required for a locus was set as 122 to 140 .

Genetic linkage map construction. We used a two-step strategy to construct two versions of the map with different genotype profiles. The first version (Version 1.0) was constructed with markers that were genotyped in more than $94 \%$ of offspring (140 individuals, missing data rate $<6 \%$, strict parameter), while the second version (Version 2.0) was constructed with markers that were genotyped in more than $85 \%$ of offspring (122 individuals, missing data rate $<18 \%$, relaxed parameter). The genotype file with low data missing rate guaranteed the high quality of the map, whereas genotype file with high data missing rate was used to increase the marker density of the map. Genotype file of Version 1.0 was first imported into JoinMap $4.0^{58}$ by selecting the BC1 population type. Individuals with more than $30 \%$ missing markers were excluded. Markers showing severe Mendelian segregation distortion were also discarded $\left(\chi^{2}>30, \mathrm{P}<0.01\right.$, d.f. $\left.=2\right)$. Pairwise recombination estimates and a logarithm of odds (LOD) score of 6.0 were applied to determine linkage groups. Then single markers that were not assigned to any linkage group and linkage groups with less than three markers were excluded from further analysis. Markers in each LG were ordered using the regression algorithm with the parameters of a recombination rate of less than 0.4 , a LOD value of greater than 1 , and 3 rounds of ordering (a jump threshold of 5). The genetic distance (cM) was estimated with the Kosambi function ${ }^{59}$. After the initial mapping, double-crossovers were tested using the "Genotype probabilities" function. Suspicious genotypes were replaced with missing values and then re-ordered with the corrected genotyping matrix. Finally, distorted markers that did not affect the order of the surrounding markers were added to the linkage groups since these markers are known to barely affect the estimation of recombination frequency ${ }^{26,60}$. To validate the map quality, Version 1.0 map was analyzed with Lep-Map 2.0 ${ }^{61}$. Only markers with orders consistent with that of JoinMap 4.0 were retained. Lep-Map 2.0 is capable of creating ultra-high-density linkage maps with high computational efficiency and accuracy implemented fully in JAVA. Because JoinMap 4.0 was unable to process the Version 2.0 genotyping data sets that contain larger set of markers, only Lep-Map 2.0 was adopted for grouping and ordering markers for this version. Finally, Version 2.0 map was compared to the Version 1.0 map, and markers with inconsistent orders were discarded. The final map was drawn with MapChart $2.2^{62}$.

To evaluate the map quality, heat map reflecting the recombination relationship between markers in each LG was generated with CheckMatrix ${ }^{63}$, and haplotype map mirroring the double crossover were generated by JoinMap 4.0 for each LG respectively (for Version 1.0 map only). A 6-kb sequencing library was constructed and sequenced in paired-ends to evaluate the map quality by Blast searching for neighboring markers using ustacks program (Supplemental Fig. S8). The coding of linkage groups and their corresponding relationships between Version 1.0 and Version 2.0 is shown in Supplementary Table S3. Genome coverage of the newly-built linkage 
map was estimated with the equation $c=1-e^{-2 d n / L}$, where $d$ is the average interval of markers, $n$ is the number of markers, and $L$ is the length of the linkage map estimated by $L=l *(m+1) /(m-1)(m$ is the number of markers in the linkage group $)^{64}$.

Anchoring contigs from the rose genome assemblies to the linkage map. The OB genome was recently obtained from doubled-haploid plants using single-molecule real-time sequencing, ${ }^{6,10}$. To verify the collinearity between our genetic map and the reference genomes and to place more contigs to the pseudo-chromosomes, we anchored the contigs to the linkage map using RAD-tag markers. Initially, all markers from the high density genetic map were searched on the genome assembly with $B L A S T+2.6 .0$ to integrate contig sequences $^{65}$. Full genome sequence was downloaded from Genome Database for Rosaceae (https://www.rosaceae. org). The marker sequences were blast searched against contigs with a cutoff E-value of $10^{-15}$. If a query hit one contig, the contig was assigned to the LG; if a query hit two or more contigs with a less than two-fold difference in the E-value, we did not assign any of these contigs. In cases where a contig was hit by multiple markers from different linkage groups, the contig was assigned to LGs with more than 2/3 markers. Inconsistency between the LGs and the pseudo-molecules of the assemblies was used to detect chromosome rearrangement events. Finally, total length of contigs anchored to the LGs was calculated. Collinearity information was generated using Circos software ${ }^{66}$.

To identify centromeric contigs, we aligned the 13 centromeric repeat sequences identified by Raymond et al. ${ }^{6}$ using Blast ${ }^{67}$. Gene and transposable element (TE) annotations were retrieved for both genome assemblies. We filtered out TEs classified as "putative host genes", and discarded gene annotations having their exons overlapped by TE annotations on more than $30 \%$ of their length. We also identified chloroplastic and mitochondrial contigs from Hibrand Saint-Oyant's genome by comparing them to reference sequences CM009590.1, KF753637.1, NC_037492.1, NC_032038.1, CM009589.1 and NC_018554.1. For all these features and Blast results, we computed the percentage of the sequence length they were covering (Supplementary Tables S8 and S9).

Sequence comparison with Fragaria vesca. To examine the conservation of synteny between rose and strawberry ( $F$. vesca), the marker sequences of our genetic map were searched against the genome sequences of Fragaria (v1.1 and v4.0.a1 assemblies; http://www.rosaceae.org) using the BLAST+2.6.0 program with default parameters ${ }^{65}$. Markers with low quality ratio were excluded: (1) The length of the alignment was less than $100 \mathrm{bp}$; (2) The hit was found at multiple locations on the scaffolds (potentially corresponding to repetitive elements); (3) E-value $<10^{-5}$. Alignments between rose LGs and the strawberry genome were visualized with $\operatorname{Circos}^{66}$.

\section{Data Availability}

All data supporting the results of this study are included in the manuscript and its additional files.

\section{References}

1. Cairns, T. ROSE COLLECTIONS AND TRIALS | Rose Societies. Encyclopedia of Rose Science, 682-687 (2003).

2. Zhang, Z. \& Zhu, X. Rose of China. (China Forestry Publishing House, 2006).

3. Wang, G. L. In Proceedings of the Ivth International Symposium on Rose Research and Cultivation Acta Horticulturae (ed. Pemberton, H. B.) 347-356 (International Society Horticultural Science, 2007).

4. Zhang, L. Genetic linkage mapping in tetraploid and diploid rose Ph.D thesis, Clemson University (2003).

5. Smulders, M. J. M. et al. In Wild Crop Relatives: Genomic and Breeding Resources: Plantation and Ornamental Crops (ed. Chittaranjan Kole) 243-275 (Springer Berlin Heidelberg, 2011).

6. Raymond, O. et al. The Rosa genome provides new insights into the domestication of modern roses. Nature Genetics 50, 772-777, https://doi.org/10.1038/s41588-018-0110-3 (2018).

7. Bendahmane, M., Dubois, A., Raymond, O. \& Le Bris, M. Genetics and genomics of flower initiation and development in roses. Journal of Experimental Botany 64, 847-857 (2013).

8. Martin, M., Piola, F., Chessel, D., Jay, M. \& Heizmann, P. The domestication process of the Modern Rose: genetic structure and allelic composition of the rose complex. Theoret. Appl. Genetics 102, 398-404, https://doi.org/10.1007/s001220051660 (2001).

9. Liorzou, M. et al. Nineteenth century French rose (Rosa sp.) germplasm shows a shift over time from a European to an Asian genetic background. Journal of Experimental Botany 67, 4711-4725, https://doi.org/10.1093/jxb/erw269 (2016).

10. Hibrand Saint-Oyant, L. et al. A high-quality genome sequence of Rosa chinensis to elucidate ornamental traits. Nature Plants 4, 473-484, https://doi.org/10.1038/s41477-018-0166-1 (2018).

11. Firoozabady, E., Moy, Y., Courtney-Gutterson, N. \& Robinson, K. Regeneration of Transgenic Rose (Rosa hybrida) Plants from Embryogenic Tissue. Nature Biotechnology 12, 609-613, https://doi.org/10.1038/nbt0694-609 (1994).

12. Yokoya, K., Roberts, A. V., Mottley, J., Lewis, R. \& Brandham, P. E. Nuclear DNA amounts in roses. Annals of Botany 85, 557-561, https://doi.org/10.1006/anbo.1999.1102 (2000).

13. Dong, X. et al. Genetic control of flowering time in woody plants: roses as an emerging model. Plant Diversity 39, 104-110 (2017).

14. Vergne, P. et al. Somatic embryogenesis and transformation of the diploid Rosa chinensis cv Old Blush. Plant Cell, Tissue and Organ Culture (PCTOC) 100, 73-81, https://doi.org/10.1007/s11240-009-9621-z (2009).

15. Doganlar, S., Frary, A., Daunay, M. C., Lester, R. N. \& Tanksley, S. D. Conservation of gene function in the Solanaceae as revealed by comparative mapping of domestication traits in eggplant. Genetics 161, 1713-1726 (2002).

16. Wu, J. et al. High-density genetic linkage map construction and identification of fruit-related QTLs in pear using SNP and SSR markers. Journal of Experimental Botany 65, 5771-5781, https://doi.org/10.1093/jxb/eru311 (2014).

17. Watanabe, S. et al. A Map-Based Cloning Strategy Employing a Residual Heterozygous Line Reveals that the GIGANTEA Gene Is Involved in Soybean Maturity and Flowering. Genetics 188, 395-U260, https://doi.org/10.1534/genetics.110.125062 (2011).

18. Huang, S. X. et al. Draft genome of the kiwifruit Actinidia chinensis. Nat Commun 4, 2640, https://doi.org/10.1038/ncomms3640 (2013).

19. Debener, T. \& Mattiesch, L. Construction of a genetic linkage map for roses using RAPD and AFLP markers. Theoret. Appl. Genetics 99, 891-899, https://doi.org/10.1007/s001220051310 (1999).

20. Rajapakse, S. et al. Two genetic linkage maps of tetraploid roses. Theoretical and Applied Genetics 103, 575-583, https://doi. org/10.1007/Pl00002912 (2001)

21. Zhang, L. H., Byrne, D. H., Ballard, R. E. \& Rajapakse, S. Microsatellite marker development in rose and its application in tetraploid mapping. J Am Soc Hortic Sci 131, 380-387 (2006). 
22. Gar, O. et al. An Autotetraploid Linkage Map of Rose (Rosa hybrida) Validated Using the Strawberry (Fragaria vesca) Genome Sequence. Plos One 6, e20463, https://doi.org/10.1371/journal.pone.0020463 (2011).

23. Yu, C. et al. Filling gaps with construction of a genetic linkage map in tetraploid roses. Front Plant Sci 5, 796, https://doi.org/10.3389/ fpls.2014.00796 (2015).

24. Crespel, L. et al. Mapping of qualitative and quantitative phenotypic traits in Rosa using AFLP markers. Theoretical and Applied Genetics 105, 1207-1214, https://doi.org/10.1007/s00122-002-1102-2 (2002).

25. Dugo, M. L. et al. Genetic mapping of QTLs controlling horticultural traits in diploid roses. Theoret. Appl. Genetics 111, 511-520, https://doi.org/10.1007/s00122-005-2042-4 (2005).

26. Yan, Z. et al. Construction of an integrated map of rose with AFLP, SSR, PK, RGA, RFLP, SCAR and morphological markers. Theoretical and Applied Genetics 110, 766-777, https://doi.org/10.1007/s00122-004-1903-6 (2005).

27. Oyant, L. H. S., Crespel, L., Rajapakse, S., Zhang, L. \& Foucher, F. Genetic linkage maps of rose constructed with new microsatellite markers and locating QTL controlling flowering traits. Tree Genetics \& Genomes 4, 11-23, https://doi.org/10.1007/s11295-007-00842 (2008).

28. Linde, M., Hattendorf, A., Kaufmann, H. \& Debener, T. Powdery mildew resistance in roses: QTL mapping in different environments using selective genotyping. Theoretical and Applied Genetics 113, 1081-1092, https://doi.org/10.1007/s00122-006-0367-2 (2006).

29. von Malek, B., Weber, W. E. \& Debener, T. Identification of molecular markers linked to Rdr1, a gene conferring resistance to blackspot in roses. Theoretical and Applied Genetics 101, 977-983, https://doi.org/10.1007/s001220051570 (2000).

30. Moghaddam, H. H., Leus, L., De Riek, J., Van Huylenbroeck, J. \& Van Bockstaele, E. Construction of a genetic linkage map with SSR, AFLP and morphological markers to locate QTLs controlling pathotype-specific powdery mildew resistance in diploid roses. Euphytica 184, 413-427, https://doi.org/10.1007/s10681-011-0616-6 (2012).

31. Spiller, M. et al. Towards a unified genetic map for diploid roses. Theoret. Appl. Genetics 122, 489-500, https://doi.org/10.1007/ s00122-010-1463-x (2011).

32. Bourke, P. M. et al. Partial preferential chromosome pairing is genotype dependent in tetraploid rose. Plant J 90, 330-343, https:// doi.org/10.1111/tpj.13496 (2017).

33. Vukosavljev, M. et al. High-density SNP-based genetic maps for the parents of an outcrossed and a selfed tetraploid garden rose cross, inferred from admixed progeny using the 68k rose SNP array. Hortic Res-England 3, 16052, https://doi.org/10.1038/ hortres.2016.52 (2016).

34. Davey, J. W. et al. Genome-wide genetic marker discovery and genotyping using next-generation sequencing. Nat Rev Genet 12, 499-510, https://doi.org/10.1038/nrg3012 (2011).

35. Nielsen, R., Paul, J. S., Albrechtsen, A. \& Song, Y. S. Genotype and SNP calling from next-generation sequencing data. Nat Rev Genet 12, 443-451, https://doi.org/10.1038/nrg2986 (2011).

36. Elshire, R. J. et al. A Robust, Simple Genotyping-by-Sequencing (GBS) Approach for High Diversity Species. Plos One 6, e19379, https://doi.org/10.1371/journal.pone.0019379 (2011).

37. Baird, N. A. et al. Rapid SNP Discovery and Genetic Mapping Using Sequenced RAD Markers. Plos One 3, e3376, https://doi. org/10.1371/journal.pone.0003376 (2008).

38. Peterson, B. K., Weber, J. N., Kay, E. H., Fisher, H. S. \& Hoekstra, H. E. Double Digest RADseq: An Inexpensive Method for De Novo SNP Discovery and Genotyping in Model and Non-Model Species. Plos One 7, e37135, https://doi.org/10.1371/journal. pone.0037135 (2012)

39. Yang, G. Q. et al. Development of a universal and simplified ddRAD library preparation approach for SNP discovery and genotyping in angiosperm plants. Plant Methods 12, 39, https://doi.org/10.1186/s13007-016-0139-1 (2016).

40. Yan, M. Q. et al. Genotyping-by-sequencing application on diploid rose and a resulting high-density SNP-based consensus map. Hortic Res-England 5, 17, https://doi.org/10.1038/s41438-018-0021-6 (2018).

41. Heo, M. S., Han, K., Kwon, J. K. \& Kang, B. C. Development of SNP markers using genotyping-by-sequencing for cultivar identification in rose (Rosa hybrida). Hortic Environ Biote 58, 292-302, https://doi.org/10.1007/s13580-017-0268-0 (2017).

42. Li, S. et al. Inheritance of Perpetual Blooming in Rosa chinensis 'Old Blush'. Horticultural Plant Journal 1, 108-112 (2015).

43. Channeliere, S. et al. Analysis of gene expression in rose petals using expressed sequence tags. Febs Lett 515, 35-38, https://doi. org/10.1016/S0014-5793(02)02413-4 (2002)

44. Scalliet, G. et al. Biosynthesis of the major scent components 3,5-dimethoxytoluene and 1,3,5-trimethoxybenzene by novel rose O-methyltransferases. Febs Lett 523, 113-118 (2002).

45. Scalliet, G. et al. Scent evolution in Chinese roses. P Natl Acad Sci USA 105, 5927-5932 (2008)

46. Krussmann, G. The complete book of roses. Quarterly Review of Biology (1981).

47. Li, S. et al. Comparative transcriptomics identifies patterns of selection in roses. BMC Plant Biology 18, 371, https://doi.org/10.1186/ s12870-018-1585-x (2018).

48. Edger, P. P. et al. Single-molecule sequencing and optical mapping yields an improved genome of woodland strawberry (Fragaria vesca) with chromosome-scale contiguity. Gigascience 7, 7, https://doi.org/10.1093/gigascience/gix124 (2017).

49. Tanksley, S. D. et al. High density molecular linkage maps of the tomato and potato genomes. Genetics 132, 1141-1160 (1992).

50. Sargent, D. J. et al. A genetic linkage map of microsatellite, gene-specific and morphological markers in diploid Fragaria. Theor Appl Genet 109, 1385-1391, https://doi.org/10.1007/s00122-004-1767-9 (2004).

51. Liebhard, R., Koller, B., Gianfranceschi, L. \& Gessler, C. Creating a saturated reference map for the apple (Malus x domestica Borkh.) genome. Theoretical and Applied Genetics 106, 1497-1508 (2003).

52. Guo, F. et al. Construction of a SNP-based high-density genetic map for pummelo using RAD sequencing. Tree Genetics \& Genomes 11, 11, https://doi.org/10.1007/s11295-014-0831-0 (2015).

53. Ueda, Y. \& Akimoto, S. Cross- and self-compatibility in various species of the genus Rosa. Journal of Pomology \& Horticultural Science 76, 392-395 (2001).

54. Fauré, S. et al. A molecular marker-based linkage map of diploid bananas (Musa acuminata). Theoretical and Applied Genetics 87, 517-526, https://doi.org/10.1007/bf00215098 (1993).

55. Wang, X. et al. Telomere-centric genome repatterning determines recurring chromosome number reductions during the evolution of eukaryotes. New Phytol 205, 378-389, https://doi.org/10.1111/nph.12985 (2015).

56. Doyle, J. J. \& Doyle, J. L. Genomic plant DNA preparation from fresh tissue-the CTAB method. Phytochem Bull 19, 11-15 (1987).

57. Rochette, N. C. \& Catchen, J. M. Deriving genotypes from RAD-seq short-read data using Stacks. Nat Protoc 12, 2640-2659 (2017).

58. Van Ooijen, J. JoinMap 4, Software for the calculation of genetic linkage maps in experimental populations. Kyazma BV, Wageningen, The Netherlands (2006)

59. Vinod, K. K. Kosambi and the genetic mapping function. Resonance 16, 540-550, https://doi.org/10.1007/s12045-011-0060-x (2011).

60. Bartholome, J. et al. High-resolution genetic maps of Eucalyptus improve Eucalyptus grandis genome assembly. New Phytol 206, 1283-1296, https://doi.org/10.1111/nph.13150 (2015).

61. Rastas, P., Calboli, F. C., Guo, B., Shikano, T. \& Merila, J. Construction of Ultradense Linkage Maps with Lep-MAP2: Stickleback F2 Recombinant Crosses as an Example. Genome Biol Evol 8, 78-93, https://doi.org/10.1093/gbe/evv250 (2015).

62. Voorrips, R. E. MapChart: Software for the graphical presentation of linkage maps and QTLs. J Hered 93, 77-78 (2002).

63. West, M. A. L. et al. High-density haplotyping with microarray-based expression and single feature polymorphism markers in Arabidopsis. Genome Res 16, 787-795 (2006). 
64. Remington, D. L., Whetten, R. W., Liu, B. H. \& O'Malley, D. M. Construction of an AFLP genetic map with nearly complete genome coverage in Pinus taeda. Theoretical and Applied Genetics 98, 1279-1292 (1999).

65. Camacho, C. et al. BLAST plus: architecture and applications. Bmc Bioinformatics 10 (2009).

66. Krzywinski, M. et al. Circos: An information aesthetic for comparative genomics. Genome Res 19, 1639-1645 (2009).

67. Altschul, S. F., Gish, W., Miller, W., Myers, E. W. \& Lipman, D. J. Basic Local Alignment Search Tool. Journal of Molecular Biology 215, 403-410, https://doi.org/10.1016/s0022-2836(05)80360-2 (1990).

\section{Acknowledgements}

We are grateful to Profs. De-Zhu Li, and Zhenhua Guo for offering access to high-performance computers. We thank Prof. David H. Byrne for the R. wichuraiana 'Basyes' Thornless' (BT) materials. This work was partially facilitated by the Germplasm Bank of Wild Species of China and the Pôle Scientifique de Modélisation Numérique of the ENS de Lyon-France. This work was supported by grants from National Natural Science Foundation of China (31660583, 31160402), the National High Technology R\&D Program of China (2011AA100208), the CAS Pioneer Hundred Talents Program (292015312D11035), and the Yunnan Recruitment Program of Experts in Science.

\section{Author Contributions}

J.Y.H., K.T. and S.L. designed experiments and provided supervisory roles; S.L. created the BC1F1 population, phenotyped the plants, and prepared the DNA samples; G.Y., S.Y., X.D., N.Z., H.Y., J.J., H.J., Q.W., M.C., X.Q., H.Z., X.J., Y.S., M.Z., M.B. and G.N. generated and analyzed data; G.Y. prepared the figures with the discussion from J.Y.H.; J.Y.H., S.L., G.Y. and H.G. wrote the manuscript with the help from all authors. All authors have read and approved the manuscript.

\section{Additional Information}

Supplementary information accompanies this paper at https://doi.org/10.1038/s41598-019-42428-y.

Competing Interests: The authors declare no competing interests.

Publisher's note: Springer Nature remains neutral with regard to jurisdictional claims in published maps and institutional affiliations.

(c) (i) Open Access This article is licensed under a Creative Commons Attribution 4.0 International License, which permits use, sharing, adaptation, distribution and reproduction in any medium or format, as long as you give appropriate credit to the original author(s) and the source, provide a link to the Creative Commons license, and indicate if changes were made. The images or other third party material in this article are included in the article's Creative Commons license, unless indicated otherwise in a credit line to the material. If material is not included in the article's Creative Commons license and your intended use is not permitted by statutory regulation or exceeds the permitted use, you will need to obtain permission directly from the copyright holder. To view a copy of this license, visit http://creativecommons.org/licenses/by/4.0/.

(C) The Author(s) 2019 